\title{
Mass of a Black Hole Firewall
}

\author{
M.A. Abramowicz ${ }^{1,2}$ * W. Kluźniak ${ }^{1}$ W and J.-P. Lasota ${ }^{3,1,4}$ \\ ${ }^{1}$ Copernicus Astronomical Center, ul. Bartycka 18, 00-716 Warszawa, Poland \\ ${ }^{2}$ Department of Physics, University of Gothenburg, SE-412-96 Göteborg, Sweden \\ ${ }^{3}$ IAP, UMR 7095 CNRS, UPMC Univ Paris 06, 98bis Bd Arago, 75014 Paris, France and \\ ${ }^{4}$ Astronomical Observatory, Jagiellonian University, ul. Orla 171, 30-244 Kraków, Poland
}

(Dated: July 10, 2018)

Quantum entanglement of Hawking radiation has been supposed to give rise to a Planck density "firewall" near the event horizon of old black holes. We show that Planck density firewalls are excluded by Einstein's equations for black holes of mass exceeding the Planck mass. We find an upper limit of $1 /(8 \pi M)$ to the surface density of a firewall in a Schwarzschild black hole of mass $M$, translating for astrophysical black holes into a firewall density smaller than Planck density by more than 30 orders of magnitude. A strict upper limit on the firewall density is given by the Planck density times the ratio $M_{\mathrm{Pl}} /(8 \pi M)$.

PACS numbers: 04.70.Dy, 04.70.Bw 


\section{INTRODUCTION}

Almheiri et al.[1] argue that two standard assumptions made in discussions of quantum properties of black holes, namely that "i) Hawking radiation is in a pure state, ii) the information carried by the radiation is emitted near the horizon, with low energy effective field theory valid beyond some distance from the horizon," are incompatible with a statement that iii) the infalling observer encounters nothing unusual at the horizon [2]. Their proposed "resolution [of the apparent contradiction] is that the infalling observer burns up at the horizon." Specifically, they suggest that "the infalling observer encounters a Planck density of Planck scale radiation and burns up." This phenomenon has been called a "firewall," and firewalls were supposed to be present both in stellar-mass and supermassive black holes. While the presence of a firewall is subject to an ongoing controversy (e.g., [3]), the internal contradiction of the set of three assumptions seems to be real and supported by detailed calculations in [1] and numerous other papers (e.g., [4] and references therein).

With the caveat that the final status of such calculations is subject to verification in a future theory of quantum gravity, we would like to explore some consequences of the postulated presence of a Planck density firewall. We will restrict ourselves to the Schwarzschild black hole, which guarantees spherical symmetry of the firewall, if present. As far as we are aware there is no theory of what a firewall is or what its influence on the (classical) space-time structure would be. In the present letter we address a specific aspect of this problem by calculating the gravitational mass of a Planck-density firewall in a Schwarzschild black hole. We take "Planck density" to mean a density of $M_{\mathrm{Pl}} c^{2} / l_{\mathrm{Pl}}^{3}$, with $l_{\mathrm{Pl}}=M_{\mathrm{Pl}} G / c^{2}$, and $M_{\mathrm{Pl}}=2.18 \cdot 10^{-5} \mathrm{~g}$ the Planck mass. Henceforth, we suppress factors of $G$ and $c$.

\section{MASS OF BLACK HOLE FIREWALLS}

We begin by checking whether the mass of a firewall may be neglected in the discussion of the space-time metric. The gravitational mass of a spherical shell in the presence of a Schwarzschild black hole has been examined by a number of authors (e.g., [5]), the calculations are a standard application of Einstein's equations and have never been subject to controversy. In the limit of low mass-energy of the shell, i.e., neglecting non-linear effects, its gravitational

effect is to increase the mass of the black hole, as perceived by a distant observer, by $M_{F}=E_{F} g_{t t}^{1 / 2}$, as would be expected from a simple redshift of energy. Here, $E_{F}$ is the mass of the shell in the local frame, and

$$
g_{t t}^{1 / 2}=(1-2 M / r)^{1 / 2}
$$

is the usual redshift factor associated with a black hole mass $M$ and radial Schwarzschild co-ordinate $r$. Thus, the gravitational mass of the black hole with the shell placed at $r$ is

$$
M_{t}=M+E_{F}(1-2 M / r)^{1 / 2}
$$

as long as $M \gg\left|M_{t}-M\right|$. In the firewall literature everyone seems to be assuming that the presence of the firewall would not significantly affect the space-time metric. In fact this is not the case. We begin by showing that a Planck density shell would have a non-negligible contribution to the gravitational mass of the system.

It is straightforward to compute the mass-energy of the firewall in the local rest-frame. We assume a Planck density shell of radius $r=2 M$ and thickness $\lambda$, equal to the Planck length, $\lambda=l_{\mathrm{Pl}}$. Hence,

$$
E_{F}=16 \pi M^{2} l_{\mathrm{Pl}} \frac{M_{\mathrm{Pl}}}{l_{\mathrm{Pl}}^{3}}=16 \pi M \frac{M}{M_{\mathrm{Pl}}} .
$$

The corresponding mass of the firewall, as perceived by a distant observer would be

$$
M_{F}=16 \pi M \frac{M}{M_{\mathrm{Pl}}}(1-2 M / r)^{1 / 2},
$$

as long as $M_{F} \ll M$. For definiteness we will assume that the firewall is located just a Planck proper length away from the horizon. Neglecting a factor of $1 / 3$ which would arise from averaging over the interval $r=\left(2 M, 2 M+l_{\mathrm{Pl}}\right)$, the corresponding redshift factor may be evaluated with

$$
r-2 M=\frac{1}{8 M} l_{\mathrm{Pl}}^{2},
$$


to yield the final formula

$$
M_{F}=4 \pi M .
$$

Therefore $M_{F}>M$, i.e., the firewall would have a mass exceeding the mass of the "bare" black hole, in contradiction with our assumption that $M_{F} \ll M$. A Planck density firewall cannot be treated as a negligible perturbation to the space-time structure of a black hole. Therefore, we must consider the "back reaction" of the firewall on the metric.

We will now examine constraints on the gravitational mass of a black hole and a shell of energy $E_{F}$ placed at $r$. By ref. [5] one can express the gravitational mass of the shell as seen by an observer at infinity as

$$
M_{F}=M_{t}-M=E_{F}\left[\left(1-\frac{2 M}{r}\right)^{1 / 2}-\frac{E_{F}}{2 r}\right] .
$$

This equation expresses the non-linearity of the Einstein equations [5]. It can be rewritten as

$$
\left(\frac{M_{F}}{E_{F}}\right)^{2}-\left(1-\frac{2 M}{r}\right)^{1 / 2}\left(\frac{M_{F}}{E_{F}}\right)+\frac{M_{F}}{2 r}=0
$$

with the solution

$$
2\left(\frac{M_{F}}{E_{F}}\right)=\left(1-\frac{2 M}{r}\right)^{1 / 2}+\left(1-\frac{2 M_{t}}{r}\right)^{1 / 2} .
$$

For $M_{t}=M$ this reduces to Eq. (2). However, with the shell placed at $r=2 M_{t}$ the equations require $M_{t}>M$. Indeed, for $r=2 M_{t}$, Eq. (6) reads

$$
\left(\sqrt{M_{F} M_{t}}-E_{F} / 2\right)^{2}=0
$$

with the solution

$$
M_{t}=\left(M+\sqrt{M^{2}+E_{F}^{2}}\right) / 2 .
$$

Note that $M_{t}$ does not depend on the sign of $E_{F}$. For a fixed value of $E_{F}$, this would be the final expression for the total mass of the shell and the black hole. In particular, one would have $M_{t}-M \gg M$ for $E_{F} \gg M$. However, the real difficulty with the firewall is that its energy is proportional to the area of the horizon, i.e., to the square of the black hole mass, whereas Eq. (10) tolerates at most the first power of $M_{t}$ in the limit of large $E_{F}$. For a Planck density shell at $r=2 M_{t}$

$$
E_{F}=16 \pi \frac{M_{t}^{2}}{M_{\mathrm{Pl}}}
$$

and this makes Eq. (10) equivalent to

$$
M_{t}^{2}=\frac{M_{\mathrm{Pl}}^{2}}{(8 \pi)^{2}}\left(1-\frac{M}{M_{t}}\right)
$$

yielding an upper limit to the total mass of the black hole with a Planck density firewall at the horizon

$$
M_{t}<\frac{M_{\mathrm{Pl}}}{8 \pi} \text {. }
$$

We conclude that a firewall of Planck density placed at the horizon of a Schwarzschild black hole is not a solution to Einstein's equations for a black hole of mass exceeding the Planck mass.

\section{DISCUSSION}

Our calculations were based on the assumption of a Planck density shell in a static observer's frame. To complete our discussion of the firewall mass we must examine alternate possibilities to the one just considered. These are

a) the firewall contains negative energy states,

b) the Planck density is measured in the frame of an observer falling in from infinity,

c) the firewall density is substantially smaller than the Planck density. 


\section{Negative energy states}

Quantum states can violate the weak energy condition so one may be tempted to take $M_{F}<0$. Survival of the black hole could then require considerable fine tuning. Indeed, since $\left|M_{F}\right| \gg M$ in Eq. 4 , one may worry that a negative value of $E_{F}$ could lead to an instant disappearance of the black hole. We can venture no opinion on the fate of an observer encoutering a negative energy firewall. Does the observer "burn up" or suffer hypothermia? However, these speculations may be unfounded. Bekenstein [6] argues that negative energy states are associated with positive mass. If this is the case, our results of Eq. (10), and following, remain unchanged in the presence of negative energy states. Such a conclusion would be consistent with the fact that $M_{t}$ in Eq. (10) depends on the square of $E_{F}$.

\section{Observer falling in from infinity}

If it is an infalling observer that encounters a Planck density firewall, the energy of the firewall seen by a static observer would be reduced by the appropriate Lorentz factor. However, this would not lead to a decrease of the mass of the firewall observed at infinity, $M_{F}$, which, as we have seen, is the firewall energy $E_{F}$ suppressed by an appropriate redshift related to the wall thickness-because of Lorentz contraction, the suppression would be weaker if it is an infalling observer that encounters the firewall, as we now show.

Consider an observer freely falling from rest at infinity. The conserved energy (of the infalling observer) per unit mass is $E=u_{t}=g_{t t} u^{t}=1$, implying that the Lorentz factor associated with the motion is $\Gamma=g_{t t}^{-1}$. If the firewall has Planck density $\left(l_{\mathrm{Pl}}^{-2}\right)$ and thickness $\lambda_{0}$ in the infalling oberver's frame, a static observer at the same location would report a density of $\left(\Gamma l_{\mathrm{Pl}}\right)^{-2}$ and thickness $\Gamma \lambda_{0}$. If this is a thin shell at $r$, the firewall energy seen by the static observer would be

$$
E_{F}=4 \pi r^{2} \frac{\lambda_{0}}{\Gamma l_{\mathrm{Pl}}^{2}} .
$$

Not having at hand a quantum theory of gravity, we must assume that $\lambda_{0} \geq l_{\mathrm{Pl}}$. To minimize $E_{F}$ we take $\lambda_{0}=l_{\mathrm{Pl}}$. The relation between incremental thickness $d l_{0}$ in the infalling observer's frame, $d l$ in the static observer's frame, and the Schwarzschild radial coordinate increment is $\Gamma d l_{0}=d l=g_{t t}^{-1 / 2} d r$ so

$$
l_{\mathrm{Pl}}=\lambda_{0}=\int d l_{0}=\int g_{t t}^{1 / 2} d r=\frac{2}{3} r x^{3 / 2},
$$

where the last integration is performed from $r$ to $r(1+x)$. Taking, as before, $r=2 M$ we have the redshift factor of the shell

$$
x^{1 / 2}=\left(\frac{3 l_{\mathrm{Pl}}}{4 M}\right)^{1 / 3}
$$

and $\Gamma=x^{-1}$. Eq. (2) now gives

$$
M_{F}=16 \pi M \frac{M}{M_{\mathrm{Pl}}} x^{3 / 2}=12 \pi M,
$$

a result essentially unchanged from that of Eq. (5).

\section{Lower density firewall}

It is true that by sufficiently decreasing the density of the firewall one may avoid the difficulties discussed above. However, on dimensional grounds it is expected that as a quantum gravity effect the firewall has a Planck density, so while a lower density firewall could perhaps be made to satisfy Einstein's equations, explaining the magnitude of the density would pose a separate problem for the theory. Further, as we will now show, the firewall density cannot be universal, it has to depend on the black hole mass.

Let us allow the firewall density to be reduced by a factor of $\beta \Gamma$ with respect to the Planck density, where $\Gamma=1$ if the density is chosen in the static observer's frame, and

$$
\Gamma=\left(\frac{4 M}{3 M_{\mathrm{Pl}}}\right)^{2 / 3}
$$


if the density is chosen in the freely-falling observer's frame. Repeating the calculation of firewall mass-energy and gravitational mass for $r=2 M_{t}$ we get

$$
E_{F}=16 \pi \frac{M_{t}^{2}}{M_{\mathrm{Pl}}} \beta
$$

and

$$
M_{t}^{2}=\frac{M_{\mathrm{Pl}}^{2}}{(8 \pi \beta)^{2}}\left(1-\frac{M}{M_{t}}\right)
$$

As the firewall is supposed to arise as a result of (dis)entanglement of Hawking radiation, we assume that the gravitational mass of the black hole with the firewall should not be appreciably larger than that of the "bare" black hole. Accordingly, we take $M_{t}$ to differ from $M$ by a quantity on the order of Planck length

$$
M_{t}-M=\alpha M_{\mathrm{Pl}},
$$

with $\alpha=\mathcal{O}(1)$. Finally, we obtain

$$
\beta=\frac{\alpha^{1 / 2}}{8 \pi}\left(\frac{M_{\mathrm{Pl}}}{M}\right)^{3 / 2} \sim 0.1\left(\frac{M_{\mathrm{Pl}}}{M}\right)^{3 / 2} \ll 1 .
$$

Eq. (9) directly implies $E_{F}=2 \sqrt{\left(M_{t}-M\right) M_{t}}$. Note that this translates into a limit on the surface density of the firewall of $E_{F} /\left(16 \pi M_{t}^{2}\right)<1 /\left(8 \pi M_{t}\right)$. This is to be compared with the Planck surface density $1 / M_{\mathrm{Pl}}$. Since the thickness $\lambda$ of the shell cannot be lower than the Planck length, we arrive at a strict upper limit on the firewall density

$$
\frac{E_{F}}{16 \pi \lambda M_{t}^{2}}<\frac{1}{8 \pi}\left(\frac{M_{\mathrm{Pl}}}{M_{t}}\right) M_{\mathrm{Pl}}^{-2}
$$

i.e., the density has to be diminished with respect to the Planck density by at least the factor $M_{\mathrm{Pl}} / M_{t}$. The additional $1 / 2$ power of this factor in Eq. (20) follows from the additional requirement that the firewall contribute to the gravitational mass no more than a quantity on the order of a Planck mass.

We end this discussion with a caveat. Eq. (12) is based on the assumption that the firewall is placed just outside the horizon, as considered by some authors, e.g., 7]. After this work was completed it has been brought to our attention that "it is generally believed that if the firewall is real it is restricted to the interior." Inside the horizon $(r<2 M)$ no static structures can exist, and all geodesics end at the Schwarzschild singularity. Quite apart from the problem of the firewall energy greatly exceeding the mass of the black hole, c.f. Eq. (11), which necessitates a careful treatment of the influence of the firewall on the space-time metric, placing a steady firewall inside the horizon would give rise to the additional difficulty of the black hole mass varying in time as the firewall photons accrete onto the central singularity while the firewall is continually being recreated. A computation of the resulting rate of change of the black hole mass is outside the scope of this Letter. These difficulties could possibly be cured if the firewall existed deep inside the black hole at, say, $r<<\sqrt{M l_{\mathrm{Pl}} / 16 \pi}$.

\section{CONCLUSIONS}

We have shown that a Planck density firewall placed at the Schwarzschild horizon does not satisfy Einstein's equations for a black hole mass exceeding the Planck mass. Any shell located at the horizon of an astrophysical black hole must necessarily have a density many orders of magnitude lower than the Planck density.

We find that the density of the firewall is at most $\beta \Gamma M_{\mathrm{Pl}}^{-2}$, with $\beta$ given by Eq. (20), and $\Gamma$ by Eq. (16), its mass-energy being proportional to the geometrical mean of the Planck mass and the gravitational mass of the system $E_{F}=2 \sqrt{\alpha M_{\mathrm{Pl}} M_{t}}$. For a $10 M_{\odot}$ black hole the density works out to be $\beta \sim 10^{-59}$ Planck density if it is discussed in the static observer's frame, or $\beta \Gamma \sim\left(M_{\mathrm{Pl}} / M\right)^{5 / 6} \sim 10^{-34}$ Planck density if the density is discussed in the infalling observer's frame. These densities are considerably smaller than the ones assumed in $[1,8]$.

We are grateful to Drs. Don Marolf and Iwona Kotko for helpful comments. We thank the referees for critical remarks that helped us to improve the presentation of the results. This work was supported in part by Polish NCN 
grants 2013/08/A/ST9/00795, and 2011/01/B/ST9/05439 and by the National Science Foundation under Grant No. NSF PHY11-25915.

* marek.abramowicz@physics.gu.se

† wlodek@camk.edu.pl

* lasota@iap.fr

[1] Almheiri, A., Marolf, D., Polchinski, J., Sully, J., J. High Energy Phys. 02062 (2013)

[2] Susskind, L., Thorlacius, L., Uglum, J., Phys. Rev. D48 3743 (1993)

[3] Mathur, S.D., Turton, D., (2013) arXiv:1306.5488

[4] Brady, L., Sahakian, V., Phys. Rev. D88 600 (2013)

[5] Frauendiener, J., Hoenselaers, C., Konrad, W., Classical Quantum Grav. 7585 (1990)

[6] Bekenstein, J.D., arXiv:1310.6203v2 (2013)

[7] Hawking, S., arXiv:1401.5761 (2014)

[8] Braunstein, S.L., Pirandola, S. and Życzkowski, K., Phys. Rev. Lett.110, 101301-1/5 (2013) 